\title{
ANÁLISIS DE LA COMPRENSIÓN DEL CONCEPTO DE PARÁBOLA EN UN CONTEXTO UNIVERSITARIO
}

\section{ANALYSIS OF THE UNDERSTANDING OF THE CONCEPT OF PARABLE IN A UNIVERSITY CONTEXT}

\author{
Jorge Hernán López-Mesa ${ }^{1}$ | Eliécer Aldana-Bermúdez² $\mid$ Adrian Alonso-Arboleda ${ }^{3}$
}

Forma de citar: LÓPEZ-MESA Jorge, ALDANA-BERMÚDEZ Eliécer, ALONSO-ARBOLEDA Adrian. Análisis de la comprensión del concepto de parábola en un contexto universitario. Respuestas. 2013; 18(2): 74-79.

Recibido:

Septiembre 18 de 2012

Aceptado:

Agosto 22 de 2013

\section{RESUMEN}

El trabajo de investigación es parte de un estudio que busca analizar cómo los estudiantes llegan a la comprensión del concepto de parábola como una cónica, y las dificultades que encuentran en la construcción de este concepto matemático, para ello se ha utilizado el marco teórico de las Situaciones Didácticas, y la metodología de la Ingeniería Didáctica, apoyada en cuestionarios, entrevista y videograbaciones, y con la utilización de entornos informáticos. A partir del análisis en su fase didáctica se muestran algunos resultados sobre los procesos cognitivos y las dificultades que presentan los alumnos en el desarrollo de la comprensión /construcción del concepto de parábola.

Palabras clave: Parábola, geogebra, sistemas de representación, situaciones didácticas, ingeniería didáctica.

\section{ABSTRACT}

The research work is part of a study that seeks to analyze how students come to the understanding of the concept of parabola a Conic, and the difficulties encountered in the construction of this mathematical concept, so has used the framework of didactic situations, and the methodology of teaching engineering, based on questionnaires, interview and recordings, and with the use of computing environments. Based on the analysis in the didactic phase are some results on cognitive processes and the difficulties posed by students in the development of understanding the concept of parabola construction.

Keywords: Parable, geogebra, systems of representation, didactic situations, didactic engineering. 


\section{INTRODUCCIÓN}

E n la comprensión de la parábola como una cónica se observan dificultades en los estudiantes, su trabajo se apoya en procesos algorítmico y memorístico, y dejan de lado la comprensión analítica del concepto, como lo plantean Santa y Jaramillo (2007), hay desconocimiento de los componentes que integran la parábola, lo que indica que no pueden relacionar sus elementos de manera analítica, tal como plantea De Alba (2010); para indicar que uno de los problemas es que los estudiantes no reconocen la parábola a partir de sus componentes y por tanto no hay relación analítica de la ecuación con los elementos que la conforman, es decir, no logran integrar la razón que existe entre el vértice, la directriz y el foco, confunden la simetría con el eje focal y la orientación del objeto; tienen dificultad para utilizar los sistemas de representación que facilitan la interpretación de la parábola; porque no asocian la ecuación con la gráfica, ni establecen una relación tabular con su gráfica. De su parte, Gómez y Carulla (2000) plantean que el tratamiento gráfico que realizan los estudiantes en la comprensión geométrica de la parábola es muy complejo por la síntesis que deben realizar en los modos de representación gráfico, algebraico y analítico. Asimismo, se presentan concepciones erróneas que imposibilitan la comprensión del concepto de lugar Geométrico, como lo plantea Fernández (2010). Es por esta razón que el presente estudio inscrito en la línea de didáctica de la matemática en pensamiento matemático avanzado plantea como pregunta de investigación ¿Cómo lograr que los estudiantes universitarios adquieran comprensión del concepto de parábola, mediante geometría dinámica?

En este sentido, con el propósito de dar respuesta a la formulación de este problema de investigación y al objetivo central de este estudio, se ha utilizado para ello, como marco teórico las situaciones didácticas de Brousseau (1986), estas actividades corresponden a un conjunto de secuencias de clase que deben estar: diseñadas, organizadas y articuladas en un periodo de tiempo por el profesor, con el fin de desarrollar un proyecto de aprendizaje y un saber constituido o en vías de construcción. Una Situación Didáctica, es entonces un proceso en el cual el docente proporciona el medio didáctico para que el estudiante construya su conocimiento, es un producto resultante de un análisis a priori y a posteriori de unas acciones puestas en práctica. Además, una situación didáctica en matemáticas contiene a la vez varios constructos teóricos: El contrato didáctico, la situación problema, la variable didáctica y la situación a-didáctica. El contrato didáctico (Chevallard, 1998), se refiere a los compromisos y a los resultados que espera el profesor del estudiante y viceversa.

La primera fase en una situación didáctica es la situación a-didáctica, en la que la intención de enseñanza no es explícita para el estudiante, no hay una intención del maestro en cuanto al saber, sólo se centra en motivar, en ayudar al sujeto en una fase de adaptación con el medio. En esta fase aparece la devolución (Brousseau, 1998): La devolución es el acto por el cual el enseñante hace aceptar al alumno la responsabilidad de una situación de aprendizaje (a-didáctica) o de un problema y él mismo acepta las consecuencias de esta transferencia.

\section{MATERIALES Y MÉTODOS}

El tipo de metodología utilizada en este estudio es cualitativa interpretativa, porque admite la comprensión (Dreyfus \& Eisenberg, 1990), (citado por Aldana, 2011), en cuanto permite explicar la forma cómo los estudiantes universitarios logran comprensión del concepto de parábola, mediante la utilización de geometría dinámica, apoyada en la metodología de la Ingeniería Didáctica (Chevallard, 1998), la cual contribuye para que los estudiantes se apropien de un saber constituido o en vías de construcción. Este estudio metodológico de investigación tiene varias fases: (a) elaboración de situaciones a-didácticas y validación por juicio de expertos, (b) aplicación de las
Junio - Diciembre 2013 ISSN 0122-820X PP: 74-79 
No. 2

Junio - Diciembre 2013 ISSN 0122-820X

PP: $74-79$ situaciones a-didácticas (c) confrontación de la validación por juicio de expertos y los resultados alcanzados por los sujetos (d) análisis a priori de la situación a-didáctica, modificación y elaboración de las situaciones didácticas, (e) aplicación de algunas situaciones didácticas, (f) análisis a posteriori; (g) realización de entrevistas. (h) triangulación de la información.

Para el estudio se tomó una muestra de 25 estudiantes de Ingeniería de Sistemas de primer semestre, algunos son repitentes, cuyas edades oscilan entre 17 y 30 años, para lo cual se estableció un contrato didáctico, la asistencia del programa Geogebra. La información se obtuvo a partir de la utilización de varios instrumentos como: cuestionarios, entrevistas, registro de observación en el aula de clase, y video grabaciones. El análisis de la información obtenida se realizó mediante la triangulación de los diferentes instrumentos utilizados.

\section{RESULTADOS Y DISCUSIÓN}

El análisis se realizó considerando conjuntamente los tres instrumentos, con el propósito de conocer la forma cómo los sujetos logran la comprensión del concepto de parábola. Para ello, se centra el análisis en primer lugar en la tarea 1 con apoyo de medios informáticos y la utilización del software Geogebra.

Tarea 1. Cuestionario 4 situaciones didácticas

Utilizando la ventana de entrada en la parte inferior de la pantalla ingrese una ecuación canónica de la parábola que cumpla los siguientes requisitos: $\mathrm{Su}$ eje focal sea el eje $\mathrm{x}$, su centro este en el origen. E Identificar los siguientes elementos

a) Según su eje focal hacia donde se orienta la parábola. ¿Explique?

b) Identificar las coordenadas del foco y del vértice

c) Escribir la ecuación de la directriz

d) Determinar la longitud del lado recto

e) Representar gráficamente la ex presión.
A continuación se observa la manera como el estudiante (E5) realiza la tarea. (Ver Figura 1)

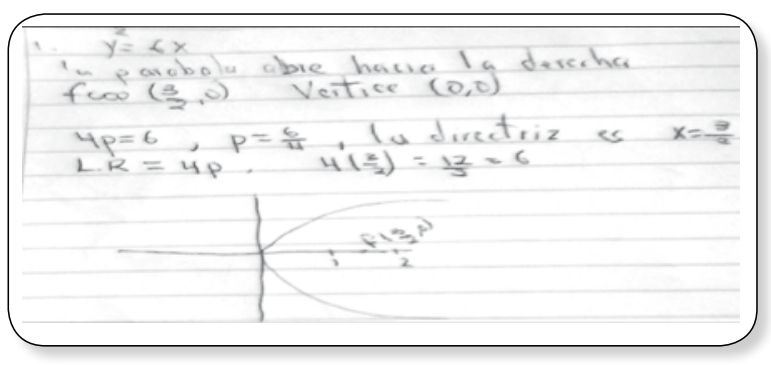

Figura 1. Desarrollo de la tarea 1, por el estudiante E5.

El estudiante digita la ecuación $\mathrm{y}^{2}=6 \mathrm{x}, \mathrm{y}$ hace al análisis en forma correcta, con la utilización algunas concepciones no apropiadas en el caso de la orientación, hace referencia a la abertura de la parábola; se puede afirmar que hay una buena correlación entre los elementos que integran a la parábola como son el vértice, el foco, el lado recto considerando la ecuación canónica; en cuanto a la representación gráfica falta información, lo cual se debe a que el alumno hizo dicha representación en el medio informático.

Durante la entrevista cuando se le pregunta, esto es lo que argumenta el estudiante.

P: ¿Cómo puedo saber que la ecuación $y^{2}=6 x$ corresponde a una parábola con vértice en el origen, y con orientación hacia la derecha?

E4: sencillo la ecuación canónica de una parábola con vértice en el origen es $y^{2}=4 p x$ y $x^{2}=4 p y$, como se pide que abra hacia la derecha entonces es $y^{2}=4 p x$. Además cuando ingreso la ecuación en el programa, se hace una gráfica como se pide.

P: ¿Qué indica el eje focal en una parábola? Explique

E4: Es el mismo eje principal, es decir, donde abre la parábola, en esta ecuación la parábola abre hacia la derecha.

P: ¿Qué relación tiene el valor de p con el eje focal?

E4: El valor de p, es la distancia que hay del 
vértice al foco o del vértice a la directriz. [se repite la pregunta] ... Ah ya, el valor de $\mathrm{p}$ es la distancia en el eje principal de la parábola.

P: ¿Qué características tiene una parábola con centro en el origen?

E4: Como lo mencione las ecuaciones canónicas de la parábola con vértice en el origen son $y^{2}=4 p x$ y $x^{2}=4 p y$, tienen una variable al cuadrado y la otra no.

P: ¿Cómo puedo identificar las coordenadas del foco, considerando el vértice, y el eje focal?

E4: fácil, el valor de p me permite determinar el valor de la distancia que hay entre el vértice y el foco, y el eje focal sirve para saber el sentido donde se mide esta distancia. [...]

El estudiante ha resuelto la tarea con un buen desempeño, porque identifica los elementos de la parábola que se referencian, tiene algunos errores de concepción porque confunde la orientación de la parábola con su abertura, y faltan procesos que permitan saber la forma como elabora las respuestas, sin embargo, existe un nivel de comprensión de la ecuación canónica $y^{2}=4 p x$. (El buen desempeño en cierta medida se debe a la validación de los resultados que arroja el sistema de cómputo, lo que permite realizar un análisis más detallado de la situación).

Tarea 3. Cuestionario 4 situaciones didácticas

En segundo lugar, esto es lo que hace el estudiante (E10) frente a una situación semejante planteada en la tarea 3 .

Ingresar una ecuación canónica de la parábola cuyo centro sea $\mathrm{C}(h, k)$, y su eje focal sea paralelo al eje $\mathrm{x}$ y determinar los siguientes elementos de la cónica:

a) Determinar las coordenadas del Foco y vértice

b) Escribir la ecuación de la directriz

c) ¿Cual es el valor del parámetro p?

d) Determinar la longitud del lado recto

e) Representar gráficamente la expresión
En la figura 2, se observa la manera como el estudiante (E10) realiza la tarea.

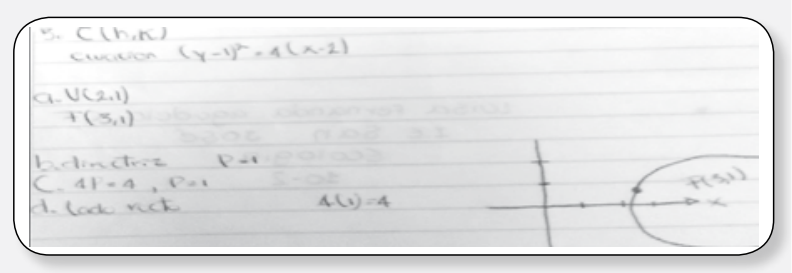

Figura 2. Desarrollo de la tarea 3, por el estudiante E10.

Se observa que el estudiante plantea como ecuación canónica que cumple con las condiciones a $(y-1)^{2}=4(x+2)$, y da solución a cada uno de los ítems en forma correcta, haciendo una relación apropiada de los elementos que integran la parábola, aunque no se muestra en forma detallada de cómo se obtienen los valores del foco, el vértice y la forma que tienen la ecuación de la directriz, sin embargo, hay una coordinación entre los valores y la relación entre ellos con respecto a la ecuación construida.

Durante la entrevista, logra identificar los elementos mediante los siguientes argumentos.

P: ¿Cómo puedo saber que la ecuación $(y+1)^{2}=4(x+2)$ corresponde a una parábola con vértice en $(h, k)$, y con orientación hacia la derecha?

E10: Esta ecuación tiene vértice en $(h, k)$, porque corresponde a la ecuación $(y-k)^{2}=4 p(x-h)$. Como y, esta al cuadrado la parábola abre a la derecha.

P: ¿Qué indica el centro C $(h, k)$ en una parábola?

E10: Indica que la parábola no tiene centro en el origen. [iCual es el vértice?] El vértice es el mismo centro $(h+p, k)$ en esta parábola. [Estudiante señala hacia la pantalla]

P: La ecuación que escribió, iconsidera el eje focal solicitado?

E10: creo que sí, porque la parábola abre hacia la derecha. Y el eje focal es $x$. 
No. 2

P: ¿Qué indica el eje focal en una parábola? Explique

E10: Es el eje principal de la parábola, nos permite conocer hacia donde abre.

P: ¿Qué relación tiene el valor de p con el eje focal?

E10: En la parábola hay una condición y es la distancia que hay del vértice al foco o del vértice a la directriz es igual. [iQué representa esta propiedad?] No entiendo

P: ¿Qué características tiene una parábola con centro fuera del origen? [...]

Durante la entrevista, el estudiante refleja un nivel de elaboración y comprensión del concepto de la ecuación canónica, porque reconoce elementos algebraicos presentes en la parábola, lo cual se comprueba en el momento con la ayuda del programa de geometría dinámica, ya que el estudiante se apoya en la visualización para dar sus respuestas y señala hacia la pantalla; y pone en evidencia una mayor comprensión del concepto de parábola mediante la utilización de estos instrumentos.

\section{CONCLUSIONES}

La metodología utilizada permite evidenciar que los estudiantes identifican las relaciones entre los elementos presentes en la representación algebraica de la ecuación canónica de la parábola, detallan el vértice, el foco, la ecuación de la directriz. Además, los registros anteriores muestran que los sujetos con la utilización de las Tics logran una mayor comprensión del objeto matemático, por la diversidad de información que tienen en la entrada gráfica y esto les permite reconocer las características presentes (Pirie, 2003). La parte visual cumple una función con estas herramientas, porque es posible ingresar datos mediante coordenadas, ecuaciones, cambiar estructuras o componentes, sin modificar el sentido de lo que se busca y pueden realizar un análisis detallado de los procesos, mediante cuestionamientos lógicos que generan comparación en el comportamiento de los datos.
El desarrollo de estructuras que permiten llegar a la construcción de un concepto es producto de la aplicación de interacciones en un medio didáctico, por ejemplo, en el caso las Tics se puede afirmar que contribuyen en esta investigación en dos sentidos, a la interiorización y comprensión del concepto; en lo actitudinal los estudiantes se muestran más receptivos y animados al desarrollo de las actividades; y en el aspecto cognitivo ponen en evidencia los procesos matemáticos que utilizan como hipótesis, conjeturas, utilización de un lenguaje matemático y abstracciones por medio de la representación gráfica; realizan la síntesis en los modos de representación algebraica y gráfica mediante la verificación y construcción de ecuaciones y la generalización a nivel de la definición del concepto, apoyadas en el concepto de lugar geométrico; esto pone de manifiesto porque son capaces de expresar con mayor facilidad sus ideas y mostrase seguros en el momento de hacer demostraciones utilizando la herramienta informática.

Una ingeniería didáctica (Chevallard, 1998) mediada por secuencias didácticas, permite conocer la forma cómo los estudiantes logran la comprensión del concepto de parábola, desde un análisis algebraico hasta llegar de forma analítica a la representación gráfica de la cónica. En las devoluciones se nota algunas concepciones erróneas que no afectan los procesos de coordinación analítica entre los modos de representación gráfico y algebraico.

Además, el medio informático como herramienta facilitó en los estudiantes la comprensión de los elementos que caracterizan la ecuación canónica de la parábola con centro en el origen y fuera de este; establecieron relaciones entre los elementos matemáticos y los modos de representación gráfico, algebraico y analítico, y lograron una construcción progresiva, ascendente, consciente y real del objeto matemático de estudio. 


\section{AGRADECIMIENTOS}

A nivel institucional, agradecemos al Programa de Ingeniería de Sistemas por su apoyo en la implementación de estas secuencias didácticas en el espacio académico de Geometría Analítica, por permitir generar la aplicación de nuevas estrategias de enseñanza. Asimismo, mi mayor reconocimiento y gratitud a los estudiantes de primer semestre académico y curricular del 2012 del Programa de Ingeniería de Sistemas, quienes se convirtieron en el motor fundamental para realizar el trabajo de campo.

\section{REFERENCIAS}

Brousseau, G. (1986): Fundamentos y métodos de la Didáctica de la Matemática, Universidad Nacional de Córdoba, Facultad de Matemática Astronomía y Física, Serie B, Trabajos de Matemática, No. 19 (versión castellana 1993).

Chevallard (1998). La transposición didáctica del saber sabio al saber ensenado. Aique Group editor. Argentina.

De Alba, G.; Et al (2010). La utilización de diferentes representaciones para facilitar los procesos de formación y desarrollo de la parábola. UACJ; UadeC, UACJ. XIII escuela de invierno en Matemática educativa. Memoria. México.

Dreyfus, T. y Eisenberg, T. (1990). On difficulties with diagrams: Theoretical issues. Proceedings of the four teenth International Conference for the Psychology of Mathematics Education, 2, 27 - 33.

Lyndon Martin and Susan Pirie, (2003). Making Images And Noticing Properties: The Role Of Graphing Software In Mathematical Generalisation. University of British Columbia. Vol. 15, No. 2, 171-186.

Santa, Z. et al (2007). Construcción de las secciones cónicas mediante el doblado de papel en el marco del modelo educativo de van hiele. 10 encuentro colombiano de matemáticas Educativa. Universidad de Antioquia.

Fernández M. (2010). Estudio de una Secuencia de Situaciones para la Enseñanza de las Cónicas integrando Cabri Géomètre II Plus. Profesor del Área de Educación Matemática, Departamento de Matemáticas y Estadística, Universidad de Nariño, Pasto Colombia.

Gómez y Carulla (2000). Enseñanza sobre la Función Cuadrática. Universidad de los Andes. Colombia. 